\title{
Evangélicos e voto legislativo: diversidade confessional e voto em deputados da bancada evangélica no Brasil
}

\author{
18 de janeiro de 2018
}

\section{Notas metodológicas}

Este documento reune a descrição das principais variáveis utilizadas no estudo "Evangélicos e voto legislativo: diversidade confessional e voto em deputados da bancada evangélica no Brasil". O objetivo consiste em apresentar em maior detalhe o processo de seleção de casos, construção dos indicadores e estrutura da base de dados utilizada pela pesquisa.

\section{Dados e Fontes}

\section{Dados e indicadores}

Os dados utilizados neste trabalho correspondem à informações sobre a composição da bancada evangélica na Câmara dos Deputados no Brasil durante a legislatura de 2015 a 2018. Tais informações são complementadas pela votação agregada em cada município em deputados pertencentes à bancada evangélica e outros deputados. Portanto, nossas variáveis dependentes são (1) a soma do total de votos recebidos pelos deputados membros da bancada evangélica no município dividida pelo total de votos nominais; e (2) a soma do total de votos recebidos pelos outros deputados eleitos (não membros da bancada evangélica) dividida pelo total de votos nominais.

Cada uma dessas variáveis serve como instrumento empírico para verificar, em primeiro lugar, que existe um efeito positivo da diversidade confessional no campo evangélico sobre o desempenho agregado dos deputados evangélicos eleitos e, em segundo lugar, rejeitar a hipótese alternativa de que seu efeito, caso significativo, fosse compartilhado por outros deputados (não membros da bancada evangélica). Este teste garante maior robustez dos resultados obtidos.

Os datos eleitorais também foram utilizados para calcular outros indicadores empregados no modelo relacionados ao sistema partidário, como a magnitude do distrito, a fragmentação eleitoral na eleição de 2010 para deputado federal ou a porcentagem de votos em partidos pequenos (nanicos).

A informação demográfica extraida do censo de 2010 foi fundamental para realizar o teste proposto no presente trabalho. A principal variável explicativa foi denominada aqui como diversidade confessional. É necessário deixar claro que consideramos aqui a diversidade confessional não em termos substantivos. Não se trata de entrar em questões de cáriz teológico ou nas possíveis variantes organizativas ou de rito entre as diferentes igrejas evangélicas. Consideramos aqui como diversidade a simples diferenciação nominal entre organizações que se inscrevem dentro do campo evangélico.

Esta variável explicativa foi operacionalizada de duas formas alternativas. A primeira consistiu em considerar o número total de denominações evangélicas com, pelo menos, um fiél no município. Portanto, corresponde a um indicador bruto ou não ponderado da diversidade confessional dentro do campo evangélico no município. Nesse sentido, quanto maior o número total de igrejas, maior a diversidade confessional. A segunda forma de operacionalização (que detalharemos mais abaixo) foi ponderar o número total de igrejas pelo número de fiéis que estas possuem no município. Desse modo, ainda que existam, por exemplo, dez igrejas no município, mas no qual 99\% dos fiéis se concentram em uma só, o valor ponderado se aproximará a um e não de dez, como seria o caso da primeira versão. Esse procedimento de criação de duas medidas empíricas para medir o mesmo fenômeno serve para, uma vez mais, testar a robustez da explicação e dos modelos.

O número (total e ponderado) de denominações evangélicas no município foi controlado por um conjunto de outros indicadores sociais comumente utilizados neste tipo de estudo. O primeiro, e mais evidente deles, é 
a proporção de pessoas que se autodenominam evangélicos no total da população. Como o artigo trata da representação eleitoral de um grupo demográfico específico, é de sentido comum esperar que a maior presença desse setor na população, maior o voto nos deputados que foram elegidos justamente por representar tais grupos. A inclusão dessa variável no modelo também serve de controle ou contraste à hipótese de que a diversidade importa.

Adicionalmente, foram utilizados como controle a população do município e a urbanização. Parte da literatura argumenta que o fenômeno da expansão dos evangélicos no Brasil esteve concentrado predominantemente nas grandes cidades e nas áreas mais urbanizadas. A inclusão desses indicadores busca verificar se o efeito considerado não se dilui quando estas variáveis são incorporadas. A mesma relação justifica a inclusão do Índice de Desenvolvimento Humano Municipal (IDH-M) do Programa das Nações Unidas para o Desenvolvimento (PNUD). Esta variável serve para controlar por fatores socioeconômicos, como a renda, a expectativa de vida e educação média dos municípios.

\section{Fontes de informação}

Para a confecção da base de dados, utilizamos quatro fontes de informação. A primeira foi o repositório de dados eleitorais do Tribunal Superior Eleitoral, que contém todos os resultados eleitorais, assim como os dados sobre os candidatos. A composição da bancada evangélica foi obtida diretamente da publicação "A radiografia do novo Congresso" lançada no primeiro ano de cada legislatura pelo Departamento Intersindical de Assessoria Parlamentar (DIAP). Os dados demográficos foram extraídos do Censo Demográfico de 2010 publicado pelo Instituto Brasileiro de Geografia e Estatística (IBGE). Finalmente, a informação relativa ao desenvolvimento humano municipal foi obtida por meio do Atlas do Desenvolvimento Humano no Brasil publicado pelo Programa das Nações Unidas para o Desenvolvimento (PNUD) em 2013.

3. Lista de candidatos selecionados

A tabela abaixo contém uma lista de todos os candidatos membros da bancada evangélica que foram utilizados no estudo. Os dados incluem o nome do candidato, o partido ao qual pertencia no momento da eleição e a Igreja evangélica que representa.

\begin{tabular}{lll}
\hline Candidato & Partido & Igreja \\
\hline ALAN RICK MIRANDA & PRB & Assembleia de Deus \\
JOÃO HENRIQUE HOLANDA CALDAS & SD & Igreja Internacional da Graça de Deus \\
ANDRÉ DOS SANTOS ABDON & PRB & Assembleia de Deus \\
SILAS CAMARA & PSD & Assembleia de Deus \\
SERGIO LUIS LACERDA BRITO & PSD & Batista \\
ERONILDES VASCONCELOS CARVALHO & PRB & Iurd \\
MÁRCIO CARLOS MARINHO & PRB & Iurd \\
ANTONIO LAZARO SILVA & PSC & Batista \\
ERIVELTON LIMA SANTANA & PSC & Assembleia de Deus \\
RONALDO MANCHADO MARTINS & PRB & Iurd \\
RONALDO FONSECA DE SOUZA & Pros & Assembleia de Deus \\
ANTONIO SERGIO ALVES VIDIGAL & PDT & Batista \\
CARLOS HUMBERTO MANNATO & SD & Cristã Maranata \\
MAX FREITAS MAURO FILHO & PSDB & Presbiteriana \\
JOAO CAMPOS DE ARAUJO & PSDB & Assembleia de Deus \\
FÁBIO FERNANDES DE SOUSA & PSDB & Fonte da Vida \\
CLEBER VERDE CORDEIRO MENDES & PRB & Congregação Cristã \\
ELIZIANE PEREIRA GAMA FERREIRA & PPS & Assembleia de Deus \\
VICTÓRIO GALLI FILHO & PSC & Assembleia de Deus \\
FABIO PAULINO GARCIA & PSB & Sara Nossa Terra \\
LINCOLN DINIZ PORTELA & PR & Batista \\
GEORGE HILTON DOS SANTOS CECILIO & PRB & Iurd \\
LEONARDO LEMOS BARROS QUINTÃO & PMDB & Presbiteriana \\
STEFANO AGUIAR DOS SANTOS & PSB & Evangelho Quadrangular
\end{tabular}




\begin{tabular}{|c|c|c|}
\hline Candidato & Partido & Igreja \\
\hline JOSUÉ BENGTSON & PTB & Evangelho Quadrangular \\
\hline JULIA MARIA GODINHO DA CRUZ MARINHO & PSC & Assembleia de Deus \\
\hline AGUINALDO VELLOSO BORGES RIBEIRO & $\mathrm{PP}$ & Batista \\
\hline EDMAR DE SOUZA ARRUDA & PSC & Presbiteriana Independente \\
\hline HIDEKAZU TAKAYAMA & PSC & Assembleia de Deus \\
\hline FERNANDO DESTITO FRANCISCHINI & SD & Assembleia de Deus \\
\hline ANTONIO WANDSCHEER & $\mathrm{PT}$ & Assembleia de Deus \\
\hline CHRISTIANE DE SOUZA YARED & $\mathrm{PTN}$ & Catedral do Reino de Deus \\
\hline ANDERSON FERREIRA RODRIGUES & PR & Assembleia de Deus \\
\hline FRANCISCO EURICO DA SILVA & PSB & Assembleia de Deus \\
\hline REJANE RIBEIRO SOUSA DIAS & $\mathrm{PT}$ & Batista \\
\hline AUREO LIDIO MOREIRA RIBEIRO & $\mathrm{SD}$ & Metodista \\
\hline EZEQUIEL CORTAZ TEIXEIRA & SD & Projeto Vida Nova \\
\hline BENEVENUTO DACIOLO FONSECA DOS SANTOS & PSol & Batista \\
\hline BENEDITA SOUZA DA SILVA SAMPAIO & $\mathrm{PT}$ & Assembleia de Deus \\
\hline ROSANGELA DE SOUZA GOMES & PRB & Iurd \\
\hline EDUARDO COSENTINO DA CUNHA & PMDB & Sara Nossa Terra \\
\hline WASHINGTON REIS DE OLIVEIRA & PMDB & Nova Vida \\
\hline AROLDE DE OLIVEIRA & PSD & Batista \\
\hline SÓSTENES SILVA CAVALCANTE & PSD & Assembleia de Deus \\
\hline ALTINEU CORTES FREITAS COUTINHO & $\mathrm{PR}$ & Assembleia de Deus \\
\hline CLARISSA BARROS ASSED MATHEUS DE OLIVEIRA & $\mathrm{PR}$ & Presbiteriana \\
\hline FRANCISCO FLORIANO DE SOUSA SILVA & $\mathrm{PR}$ & Mundial do Poder de Deus \\
\hline ROBERTO DA SILVA SALES & $\mathrm{PRB}$ & Iurd \\
\hline ANTÔNIO JÁCOME DE LIMA JUNIOR & $\mathrm{PMN}$ & Assembleia de Deus \\
\hline ONYX DORNELLES LORENZONI & DEM & Luterana \\
\hline ANTONIO CARLOS GOMES DA SILVA & PRB & Iurd \\
\hline RONALDO NOGUEIRA DE OLIVEIRA & PTB & Assembleia de Deus \\
\hline LINDOMAR BARBOSA ALVES & PMDB & Assembleia de Deus \\
\hline MARCOS ROGERIO DA SILVA BRITO & PDT & Assembleia de Deus \\
\hline NILTON BALBINO & PTB & Assembleia de Deus \\
\hline CARLOS AUGUSTO ANDRADE SILVA & PHS & Assembleia de Deus \\
\hline JOHNATHAN PEREIRA DE JESUS & $\mathrm{PRB}$ & Iurd \\
\hline GEOVANIA DE SA RODRIGUES & PSDB & Assembleia de Deus \\
\hline MARCO ANTONIO FELICIANO & PSC & Assembleia de Deus \\
\hline EDUARDO NANTES BOLSONARO & $\mathrm{PSC}$ & Batista \\
\hline GILBERTO NASCIMENTO SILVA & $\mathrm{PSC}$ & Assembleia de Deus \\
\hline PAULO ROBERTO FREIRE DA COSTA & $\mathrm{PR}$ & Assembleia de Deus \\
\hline ROBERTO ALVES DE LUCENA & PV & O Brasil para Cristo \\
\hline ANTONIO CARLOS MARTINS DE BULHÕES & PRB & Iurd \\
\hline BENEDITO ROBERTO ALVES FERREIRA & PRB & Iurd \\
\hline VINICIUS RAPOZO DE CARVALHO & $\mathrm{PRB}$ & Iurd \\
\hline BRUNA DIAS FURLAN & PSDB & Congregação Cristã \\
\hline JORGE TADEU MUDALEN & DEM & Igreja Internacional da Graça de Deus \\
\hline EDSON EDINHO COELHO ARAUJO & PMDB & Presbiteriana \\
\hline JOSE OLÍMPIO SILVEIRA MORAES & $\mathrm{PP}$ & Mundial do Poder de Deus \\
\hline JEFFERSON ALVES DE CAMPOS & PSD & Evangelho Quadrangular \\
\hline LAÉRCIO JOSÉ DE OLIVEIRA & $\mathrm{SD}$ & Presbiteriana \\
\hline JONY MARCOS DE SOUZA ARAÚJO & PRB & Iurd \\
\hline
\end{tabular}

4. Correlação entre as variáveis do estudo 
O gráfico abaixo contém o correlograma que representa o grau de associação entre as variáveis utilizadas neste estudo.

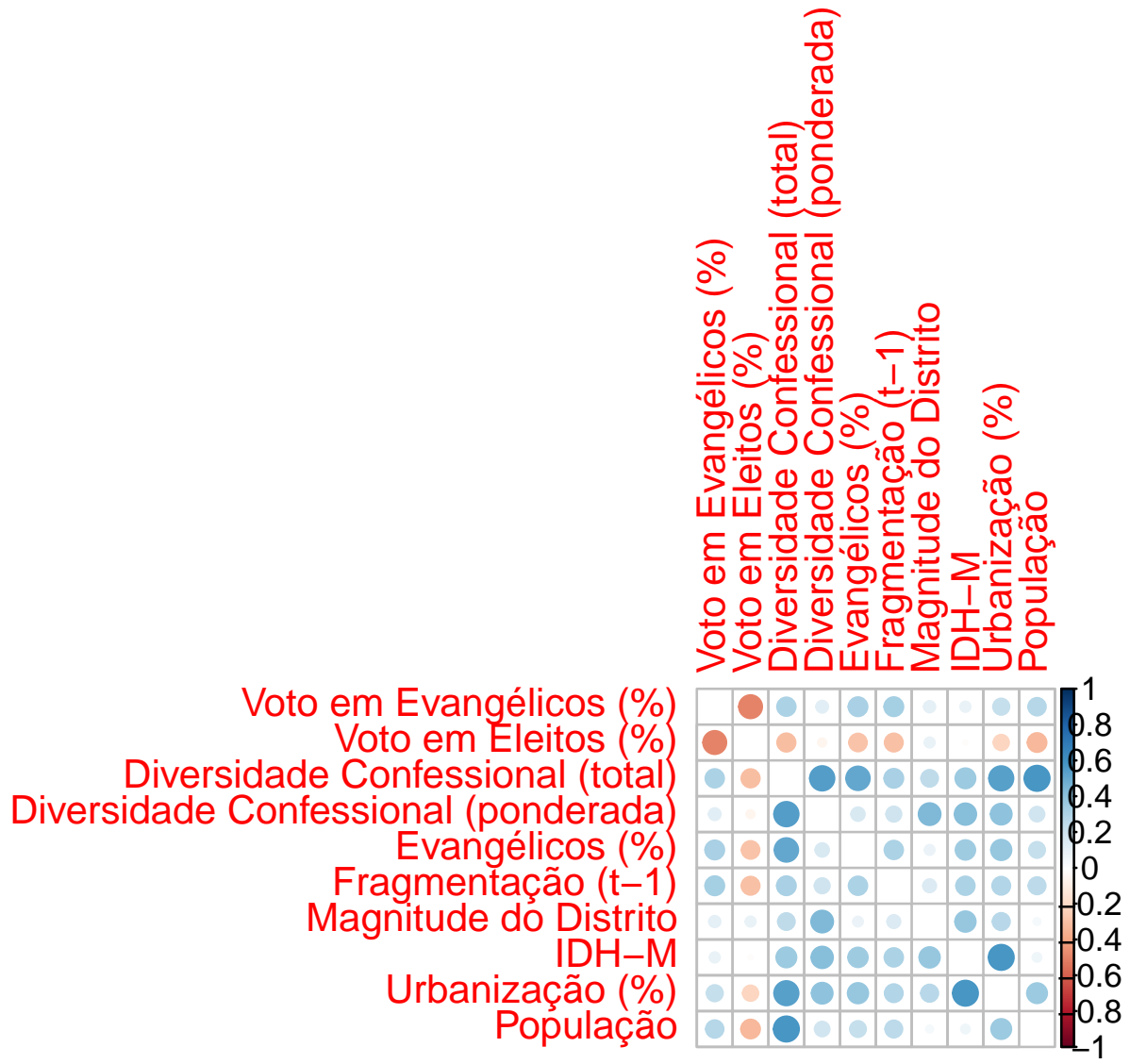




\section{Descrição detalhada das Variáveis}

\section{Porcentagem de votos em deputados evangélicos eleitos}

\section{Nome da variável: PROP_EVANG}

Corresponde à soma do total de votos obtidos por todos os deputados membros da bancada evangélica na Câmara de Deputados do Brasil na legislatura 2015-2018 dividida pelo total de votos nominais à deputado federal no município.

\section{fórmula}

$$
e v_{i} / \sum\left(v_{i}\right)
$$

onde:

ev corresponde à soma de votos obtida por todos os deputados membros da bancada evangélica que obtiveram voto no município i.

$\mathrm{v}_{\mathrm{i}}$ corresponde à soma total de votos nominais à deputado federal no município nas eleições à deputado federal em 2014.

\begin{tabular}{lllllllll}
\hline $\mathrm{N}$ & NAs & Min & Max & Range & Median & Mean & Std.Dev. & Coef.Var \\
\hline 5,570 & 0 & 0 & 0.6 & 0.6 & 0.03 & 0.06 & 0.07 & 1.34 \\
\hline
\end{tabular}

\section{Porcentagem de votos em deputados NÃO evangélicos eleitos}

\section{Nome da variável: PROP_ELEITOS}

Corresponde à soma do total de votos obtidos por todos os deputados que não fazem parte da bancada evangélica na Câmara de Deputados do Brasil na legislatura 2015-2018 dividida pelo total de votos nominais à deputado federal no município.

\section{fórmula}

$$
e l_{i} / \sum\left(v_{i}\right)
$$

onde:

$\mathrm{el}_{\mathrm{i}}$ corresponde à soma de votos obtida por todos os deputados federais que não fazem parte da bancada evangélica que obtiveram voto no município i.

$\mathrm{v}_{\mathrm{i}}$ corresponde à soma total de votos nominais à deputado federal no município nas eleições à deputado federal em 2014.

\begin{tabular}{lllllllll}
\hline $\mathrm{N}$ & NAs & Min & Max & Range & Median & Mean & Std.Dev. & Coef.Var \\
\hline 5,570 & 0 & 0.03 & 0.93 & 0.9 & 0.65 & 0.62 & 0.18 & 0.29 \\
\hline
\end{tabular}

\section{Diversidade confessional (total)}

Nome da variável: tot_igrejas 
Este indicador mede a presença das igrejas evangélicas no múnicípio, sem pondera-la pelo peso de seus fiéis. Corresponde ao número total de igrejas evangélicas no município com, pelo menos um fiel.

\begin{tabular}{lllllllll}
\hline $\mathrm{N}$ & NAs & Min & Max & Range & Median & Mean & Std.Dev. & Coef.Var \\
\hline 5,565 & 5 & 0 & 12 & 12 & 6 & 6 & 2 & 0 \\
\hline
\end{tabular}

\section{Diversidade confessional (ponderada)}

\section{Nome da variável: hh_ev}

Esta variável mede o número ponderado de igrejas segundo a distribuição de fiéis entre elas. Imaginemos um caso hipotético no qual existem quatro igrejas com cinco fiéis cada uma. Neste cenário, o número total de igrejas seria igual a quatro e o número ponderado também seria igual a quatro, pois todas possuem o mesmo número de fiéis. No entanto, se o caso fosse que que a primeira tivesse 17 e as outras um fiél cada uma, teriamos um número ponderado de 1,4 igrejas no município.

Se calcula a partir do Índice de Hirschman-Herfindahl de concentração (o mesmo utilizado para o número efetivo de partidos). Corresponde a um dividido pela soma das proporções de fiéis de cada igreja no total de evangélicos do município.

\section{fórmula}

$$
1 / \sum\left(d_{i}^{2}\right)
$$

onde:

$\mathrm{d}_{\mathrm{i}}$ corresponde à proporção de fiéis de cada igreja evangélica no município.

\begin{tabular}{lllllllll}
\hline $\mathrm{N}$ & $\mathrm{NAs}$ & Min & Max & Range & Median & Mean & Std.Dev. & Coef.Var \\
\hline 5,565 & 5 & 0 & 6.3 & 6.3 & 2 & 2.11 & 0.81 & 0.38 \\
\hline
\end{tabular}

\section{Índice de Fragmentação Eleitoral (f)}

Nome da variável: $\mathbf{f}$

\section{a. definição}

Esta variável consiste em uma medida de fragmentação do sistema partidário no nível local. Também corresponde a uma derivação do Índice de Herfindahl-Hirschman. Representa a proporção do voto não explicada pelos maiores partidos. Enquanto o Número Efetivo de Partidos Eleitorais focaliza no tamanho da concentração, este indicador revela o inverso: a importância dos votos não explicada pela dominância de grandes partidos no jogo eleitoral.

\section{b. métodos de operacionalização empírica}

Este indicador está composto por 1 menos a soma dos quadrados das proporções de voto em cada partido para deputado federal nas eleicoes de 2014. Quanto maior o valor, mais competitivo e menos concentrado é o voto. Os valores possuem um intervalo que vai de 0 a 1. 
c. fórmula

$$
1-\sum_{i=1}^{n} p_{i}^{2}
$$

Onde $p_{i}^{\wedge} 2$ é a proporção ao quadrado do voto em cada partido no município.

\begin{tabular}{lllllllll}
\hline $\mathrm{N}$ & NAs & Min & Max & Range & Median & Mean & Std.Dev. & Coef.Var \\
\hline 5,567 & 3 & 0.187 & 0.931 & 0.745 & 0.778 & 0.761 & 0.09 & 0.118 \\
\hline
\end{tabular}

\section{Magnitude do distrito}

Nome da variável: $\mathrm{dm}$

Corresponde ao número de deputados que a Unidade Federativa à que pertence o município pode eleger à Câmara de Deputados.

\begin{tabular}{lr}
\hline $\mathrm{UF}$ & $\mathrm{dm}$ \\
\hline $\mathrm{AC}$ & 8 \\
$\mathrm{AL}$ & 9 \\
$\mathrm{AM}$ & 8 \\
$\mathrm{AP}$ & 8 \\
$\mathrm{BA}$ & 39 \\
$\mathrm{CE}$ & 22 \\
$\mathrm{DF}$ & 8 \\
$\mathrm{ES}$ & 10 \\
$\mathrm{GO}$ & 17 \\
$\mathrm{MA}$ & 18 \\
$\mathrm{MG}$ & 53 \\
$\mathrm{MS}$ & 8 \\
$\mathrm{MT}$ & 8 \\
$\mathrm{PA}$ & 17 \\
$\mathrm{~PB}$ & 12 \\
$\mathrm{PE}$ & 25 \\
$\mathrm{PI}$ & 10 \\
$\mathrm{PR}$ & 30 \\
$\mathrm{RJ}$ & 46 \\
$\mathrm{RN}$ & 8 \\
$\mathrm{RO}$ & 8 \\
$\mathrm{RR}$ & 8 \\
$\mathrm{RS}$ & 31 \\
$\mathrm{SC}$ & 16 \\
$\mathrm{SE}$ & 8 \\
$\mathrm{SP}$ & 70 \\
$\mathrm{TO}$ & 8 \\
\hline &
\end{tabular}

\section{Índice de Desenvolvimento Humano Municipal}

Nome da variável: IDHM 


\section{a. definição}

Trata-se do índice de desenvolvimento humano medido no nível municipal pelo PNUD.

\begin{tabular}{lllllllll}
\hline $\mathrm{N}$ & NAs & Min & Max & Range & Median & Mean & Std.Dev. & Coef.Var \\
\hline 5,565 & 5 & 0.418 & 0.862 & 0.444 & 0.665 & 0.659 & 0.072 & 0.109 \\
\hline
\end{tabular}

\section{Taxa de Urbanização}

nome da variável: urb_rate

Proporção da população do município vivendo em áreas urbanas, segundo a classificação do IBGE.

\begin{tabular}{lllllllll}
\hline $\mathrm{N}$ & $\mathrm{NAs}$ & Min & Max & Range & Median & Mean & Std.Dev. & Coef.Var \\
\hline 5,565 & 5 & 4.2 & 100 & 95.8 & 64.7 & 63.8 & 22 & 0.3 \\
\hline
\end{tabular}

\section{População}

nome da variável: tot_pop

Total da população do município, segundo as estimativas da população para 2014 do IBGE.

\begin{tabular}{lllllllll}
\hline $\mathrm{N}$ & NAs & Min & Max & Range & Median & Mean & Std.Dev. & Coef.Var \\
\hline 5,565 & 5 & 805 & 11253503 & 11252698 & 10934 & 34278 & 203113 & 6 \\
\hline
\end{tabular}

